\title{
Summative Stereoscopic Image Compression using Arithmetic Coding
}

\author{
Thafseela Koya Poolakkachalil ${ }^{1}$, Saravanan Chandran² \\ ${ }^{1,2}$ National Institute of Technology, Durgapur, India
}

\begin{tabular}{l} 
Article Info \\
\hline Article history: \\
Received Oct 10, 2018 \\
Revised Apr 29, 2019 \\
Accepted Aug 23, 2019 \\
\hline Keyword: \\
Stereoscopic image \\
Image compression \\
Lossy compression \\
Quantization \\
Compression ratio
\end{tabular}

Thafseela Koya Poolakkachalil, National Institute of Technology, Durgapur, India

Email: thafseelariyas@hotmail.com

\begin{abstract}
Image compression targets at reducing the amount of bits required for image representation to save storage space and speed up the transmission over network. The reduction of size helps to store more images in the disk and take less transfer time in the data network. Stereoscopic image refers to a threedimensional (3D) image that is perceived by the human brain as the transformation of two images that are being sent to the left and right human eyes with distinct phases. However, storing of these images takes space twice than a single image and hence the motivation for this novel approach called Summative Stereoscopic Image Compression using Arithmetic Coding (S2ICAC) where the difference and average of these stereo pair images are calculated, quantized in the case of lossy approach and unquantized in the case of lossless approach, and arithmetic coding is applied. The experimental result analysis indicates that the proposed method achieves high compression ratio and high PSNR value. The proposed method is also compared with JPEG 2000 Position Based Coding Scheme (JPEG 2000 PBCS) and Stereoscopic Image Compression using Huffman Coding (SICHC). From the experimental analysis, it is observed that S2ICAC outperforms JPEG 2000 PBCS as well as SICHC.
\end{abstract}

Copyright (C) 2019 Institute of Advanced Engineering and Science. All rights reserved.

\section{INTRODUCTION}

The quantity of multimedia content produced and shared everyday through social media, e.g. Facebook, Instagram, Twitter, etc. and photo sharing platforms e.g. Flickr, Snapchat, Pinterest, etc. is rapidly increasing [1]. The depiction of an appeared scene to the human eye in the utmost realistic fashion is one of the foremost objectives of digital imaging. Although modern imaging systems try their best to deliver the appearance of the actual scene to an extent, there are shortcomings including correct lighting and depth reproduction. However, stereoscopic imaging and high dynamic range imaging are able to overcome approximate amount of these shortcomings [2].

The customary representation of depth presents in paintings, photographs, and television does not provide a realistic depiction of a sight as they rely monoscopic cues. Stereoscopy takes advantage of the situation and bestows a better perception of depth. Stereoscopic video cameras [3] and stereoscopic displays [4] have reached the shops. Stereoscopy has gained advantage in tasks like position, distance judgment, identifying objects, spatial manipulation of objects, navigation, and spatial understanding [5]. Medicine [6], military [7], entertainment, industrial computer aided design [8] and photogrammetry [8] are some application areas of stereoscopy. There has been a rapid escalation of 3D technologies and demand for attractive services like 3D television (3DTV) and Free Viewpoint Video (FVV) has risen. Few of the processes that the stereoscopic images go through in these applications include compression, communication, printing, display, restoration, segmentation, and fusion [9]. The success of these 3D application services depend upon providing high quality content [10]. However, this also brings many new concerns and challenges [11]-][13]. Sending 
stereoscopic 3D (S-3D) contents for major events like FIFA 2010 and 2014 has become the recent focus of many 3D media service providers [14]. An enormous amount of prominent films have been produced using the S-3D technology. 3D Video on Demand (VoD) services help in providing these movies to the viewers. Recent advances in hardware have also led to development of home stereoscopic television [15].

Image Stereoscopy is a rapidly growing research field. Stereoscopy is also known as threedimensional (3D) imaging. This technique exploits the representation and projections of images to create a 3D illusion, thus, stimulating human binocular vision. Stereoscopic image is the output 3-D image perceived by the human brain as the transformation of two images that is being transmitted to each eye with different phases [15]. Binocular vision works on the underlying principle that two images of a scene with slightly different phases are presented distinctly to the right and left human eye, the human brain gives the perception of 3D vision, upon their combination. The Human Vision System (HVS) is able to gather depth distances in the scene by comparing these two images. This principle has been exploited in 3D visuals, where the images with different polarization are projected in the same screen to give distinct image to each eye. When the observer uses eyeglasses that contain pair of opposite polarizing filters, opposite polarized light is blocked by each filter and the each eye can view only one image [16].

In the case of binocular stereo image, the raw data contains information which is double as that of monocular images. This indicates that the stereo pair consumes very large amount of memory. As a result, an increasing amount of the attention is given in research to stereo pair image compression techniques. Two aspects that have gained significant importance in the stereo pair image compression techniques were focused on reducing the disparity and where the residual image's similarity is coded to a monochrome image. In this approach, one view was used as a base to predict another view, and the coding of difference took place [17][24]. For the effective creation of a 3D system which has a pair of images, that was to be transferred in parallel, the video steam needs to be compressed before transmission. Adding to this an efficient coder has to be designed for stereoscopic image compression [17].

In image processing systems, transformation process is performed in color images to extract color spaces. In this proposed approach, RGB color transforms is used [25]. A matrix array is the output of the image analysis step [26].The technique of eliminating or decreasing the probability of redundant information by various encoding techniques, thus, reducing the storage requirement is known as data compression [27]. The term image compression indicates the process of reducing the size of the graphics file while at the same time maintaining the quality of image to an acceptable level. The advantages of image compression are mainly two: reduced transmission time and reduced storage requirement [28]-[30]. Although digital image compression has been a highly focused research area over several decades, there has been a recent shift to color image compression due to the enormous amount of color image applications as well as transmission and retrieval through various Internet based applications [31]-[33]. In stereoscopic image compression, the spatial redundancy that occurs among the two image pair was exploited to achieve greater compression ratio in comparison to individual compression of each frames [17].

Several research work has been undertaken for stereo pair image compression. A majority of the work was focused on the approach called as disparity compensated residual compression, in which one vision acts as a platform to predict the alternative, and the variance is encoded [17]-[34]. The most commonly used method here was the monoscopic compression of one channel using a suitable algorithm and the usage of other channel for prediction based on encoded disparity vectors or residual disparity [17]. Even though there has been extensive research on disparity compensation and coding for stereo image compression [23][24][18], the features of the residual disparity that occurs between the base platform and the alternative images, viz. leftright images have not been extensively studied. Hence, this paper focuses on the compression of residual disparity vector as well as the stereo pair. In this research article, compression ratio of the proposed new compression model for both lossless and lossy compression technique is also analyzed.

\subsection{Related Stereoscopic Image Compression Works}

Thafseela and Saravanan proposed a model for stereoscopic image compression where the dissimilarity between left and right images and the left image is encoded separately using Symbols Frequency based Image Coding (SFIC). It was observed from the results that the approach achieved 6 - $11 \%$ improvement in compression ratio. It was also observed that the decoding of the encoded data results in the synthesis of the retrieved left and right images with high compression ratio while at the same time maintaining the quality of the images. This motivated to perform stereoscopic image compression and retrieve right image from the decoded left image and disparity vector [33].

Said and Pearlman introduced a new algorithm where the explicit transmission of ordering data did not take place. This is centered on the fact that the algorithms that define the execution points are based on the results of comparisons [35]. Won-Ho et al. offered an algorithm for stereoscopic image compression. The offered algorithm had two stages. The first is Adaptive Limited Searching (ALS), wherein the limited searching 
is performed. Adaptive Directional Searching (ADS) is applied to identify the homogeneous directional property of disparity. For the comparison, ADS and ALS with reference vector was simulated and the results showed In ADS, the reduction of computational cost by half took place and the PSNR is slightly better than half of that of FS. This was possible due to the directional property of disparity vectors. The approach significantly reduced the computational load and it has maintained better performance when associated to traditional FS [36].

Dimitrios et al. proposed an approach where block-based hierarchical motion estimation initialized pixel approach was used for performance arrival at the disparity estimation. The results showed that this approach showed better performance than block-based approaches. The main advantage here is the fully transmitted depth information which are used in many applications [37]. Mi-Hyun and Kwang-Hoon proposed a new technique for preserving edges of the stereoscopic images. In the proposal, the problem of the intensitybased block disparity estimation techniques was investigated. From comparison of this approach with block matching algorithms and regularization technique, it was observed that this method was superior to others with respect to quality of the reconstructed image and the coding efficiency. This technique is applicable in high quality intermediate view reconstruction [38].

Won and Sung proposed an approach where the problem of extremely high computational complexity was investigated and applied in full search technique used for stereoscopic image coding. The Experimental results have shown that this algorithm significantly reduced computational complexity while maintaining high PSNR for the reconstructed image. The proposed algorithm was applied to 3D-TV transmission, 3D capable multimedia communication and multi-view image coding [39]. Po-Rong et al. proposed an approach where an MPEG based multiresolution 3-D stereoscopic video transmission over ATM based multi code CDMA channels over asynchronous transfer mode (ATM) networks was analyzed. The approach was observed to be successful in achieving higher compression ratio for characteristic 3-D stereoscopic image series while maintain the quality of the reconstructed 3-D image [40].

Francoys et al. proposed a new compression scheme. The key approaches in the approach was the decomposition of the left and right stereoscopic image pairs to low pass and high pass luminance components. The results showed that this approach obtained high CR and PSNR and motivated to concentrate on an approach that gives high CR and PSNR at the same time [41]. Sulthana et al. proposed an approach for image compression based on wavelet transform and adaptive arithmetic coding, which was an extension to Shapiro's EZW algorithm. In the approach, the image was decomposed into several subbands using discrete wavelet transform and used Shapiro's embedded zero tree wavelet algorithms, and also encoded using static zeroth order adaptive arithmetic coder. As a result, it observed better compression ratio and lesser coding time [42].

Khan et al. proposed an adaptive LSB substitution method using uncorrelated colorspace, increasing the property of imperceptibility while minimizing the chances of detection by the human vision system. Here, the input image was made to pass through an image scrambler, resulting in an encrypted image, preserving the privacy of image contents, and then converted to HSV color space for further processing. Experiments show that this method outperforms several state of-the-art methods [43].

Yun Liu et al. proposed Stereoscopic Image Quality Assessment Method based on Binocular Combination Saliency Model (SIQAM-BCSM) where a new 3D saliency map was developed that reduced the computational complexity and assigned appropriate weights to the image contents [44]. It was observed from the experimental results that proposed approach achieved Root Mean Squared Error (RMSE) of 9.0137. This motivated to perform summation of left image and right image of the stereopair images.

Pongskorn et al. proposed the application of discrete wavelet transform with arithmetic coding (DWTAC) where arithmetic coding was performed to the wavelet transformed image data. It was observed from the results that high quality images were retrieved upon decoding and the compression ratio was high [45]. This motivated to perform arithmetic coding in this research work.

Jayavrinda et al. proposed JPEG 2000 Position Based Coding Scheme (JPEG 2000 PBCS) based on the position of elements of the transform coefficients matrix where the unique elements were identified to reduce redundancies. It was observed from the results that the proposed approach produced higher compression ratio and higher PSNR [46]. This motivated to compare the proposed approach with PBCS.

Thafseela et al. focused on compressing stereoscopic images having a left and right view of a single scene using Huffman coding. This approach utilized the correlation between the stereoscopic left image and right image. This approach is based on the observation that the encoding of relative offset between the corresponding points in an image pair, termed as disparity, leads to better compression. In this approach, the colour space transformed left image is first encoded by the Huffman encoding and then the difference between the colour space transformed left and colour space transformed right images is also encoded by the Huffman encoding. In the final stage, left member of the image pair is synthesized by application of the Huffman decoding and inverse colour space transformation. The right member is synthesized by performing inverse colour space transformation to the summated output obtained from decoded left image and the Huffman 
decoded disparity vector. It was observed from the results that the proposed approach produced higher compression ratio and higher PSNR [47]. This motivated to compare the proposed approach with SICHC.

\subsection{Related Image Compression Works}

Miguel, Victor, Ian, Francesc, Michael, and Joan proposed reversible as well as irreversible colour transform based compression algorithm. They observed from the results that the approach obtained compression ratios similar to that of JPEG 2000. Another observation was that irreversible optimized transform outperformed the Karhunen-Loève Transform in terms of PSNR (up to $1.1 \mathrm{~dB}$ ). They also noticed that the reversible optimized transforms achieved PSNR of $0.9 \mathrm{~dB}$. This motivated to perform colour transformation in an optimized manner [48].

Shuyuan Zhiying, Xiandong, Jiantao, and Bing proposed a new coding scheme, where a $16 \times 16$ macro-block is converted into an $8 \times 8$ small-sized coefficient block. This resulted in improved interpolation for the retrieval of a compressed macro-block and better compression. The proposed algorithm was implemented for grayscale and colour image compression. They observed from the results that the proposed approach produced better compression gain when compared with the existing state-of-art methods, specifically for the colour images. This motivated to perform block-based transformation of colour images [49].

$\mathrm{Yu}-\mathrm{Chen} \mathrm{Fan}$ et al. proposed a scheme for luminance and correction based on which a chip was designed. The chip designed used the basis of 3-D Discrete Cosine Transform (3D-DCT) and cubic memory. This proved as a better choice as a solution for 3-D Multiview compression and storage research and compression [50][33]. Miguel, Victor, Ian, Francesc, Michael, and Joan proposed a reversible as well as irreversible colour transform based compression algorithm. They observed from the results that the approach obtained compression ratios similar to that of JPEG 2000. Another observation was that irreversible optimized transform outperformed the Karhunen-Loève Transform in terms of PSNR (up to $1.1 \mathrm{~dB}$ ). They also noticed that the reversible optimized transforms achieved PSNR of $0.9 \mathrm{~dB}$. This motivated to perform colour transformation in an optimized manner [48].

Jose et al. proposed an approach which encoded the error between color component predictions and actual values. From the experiments, it was observed that the algorithm is suitable in areas where adaptive logarithmical quantization can be applied for static images [51][33]. This motivated to focus on research area where the difference between the stereoscopic image pair is encoded. Wu-Lin et al. proposed a color encoding scheme in which three grayscale images are derived from the color image and then the grayscale image is further partitioned into a set of variable-sized blocks using quadtree segmentation. Experimental results proved that this approach cuts down the bit rates significantly, and at the same time maintains high PSNR ratio [31][33]. This motivated to focus research in such a way that high quality images are obtained at maximum bit rates.

Jinlei Zhang et al. projected a novel coding pattern for the compression of hyperspectral [HS] images. In this approach, the shifting of the difficulty of data decorrelation to decoder side was performed. Experimental results showed that this approach achieved high compression ratio for lossless compression of HS images with an encoder of low complexity [52][33]. This suggested to focus research on low complexity encoder. The research work completed by Pascal Peter proposed a new approach where a new colorization technique was introduced which made it possible for the high-quality reconstruction of color data. This motivated to include pixel replacement technique based on the difference between left image and right image [53] Shuyuan Zhiying, Xiandong, Jiantao, and Bing proposed a new coding scheme where a $16 \times 16$ macro-block is converted into an $8 \times 8$ small-sized coefficient block. This resulted in improved interpolation for the retrieval of a compressed macro-block and better compression. The proposed algorithm was implemented for grayscale and colour image compression. They observed from the results that the proposed approach produced better compression gain when compared with the existing state-of-art methods, specifically for the colour images. This motivated to perform block-based transformation of colour images [49]. The research work completed by Rushi Lan et al. motivated to club the color channels together while reconstructing the image [54][33].

The aim of this research article is to propose a novel compression scheme for stereoscopic image namely, Summative Stereoscopic Image Compression Using Arithmetic Coding (S2ICAC) for Color Image Compression. The following Section II describes the proposed S2ICAC compression technique. The following Section III Results and Analysis, analyses the proposed S2ICAC with lossless and lossy conditions, JPEG 2000 Position Based Coding Scheme (JPEG 2000 PBCS) [46], and Stereoscopic Image Compression using Huffman Coding (SICHC) [47]. Standard images are used in this experiment. Conclusions are drawn in section IV.

\section{RESEARCH METHOD}

Compression Ratio (CR) and Peak Signal to Noise ratio (PSNR) are the two main factors that measure performance of the compression scheme. While CR gives indication of the rate of compression, PSNR indicates the quality of the reconstructed image. 
a) Compression Ratio

In the case of stereoscopic images, $\mathrm{CR}$ is defined as the ratio between the added size of original left and right image $(\mathrm{n} 11+\mathrm{n} 1 \mathrm{r})$ and the added size of the compressed image $(\mathrm{n} 21+\mathrm{n} 2 \mathrm{r})$ [55].

b) Peak Signal to Noise Ratio(PSNR)

$$
C R=(n 1+n 2) /(n 2 l+n 2 r)
$$

PSNR refers to an engineering term that defines the ratio between the maximum possible power of a signal or an image and the power of corrupting noise or image that affects the quality of its representation [33].The mathematical representation of the PSNR is as follows:

$$
P S N R=20 \log _{10}\left(\frac{M A X_{f}}{\sqrt{M S E}}\right)
$$

where, MSE (Mean Square Error). MSEl and MSEr. are calculated separately for left image and right image using equation (3) and is fed into equation (2) to obtain PSNRl and PSNRr.

$$
M S E=\frac{1}{m n} \sum_{0}^{m-1} \sum_{0}^{n-1}\|f(i, j)-g(i, j)\|^{2}
$$

Image compression can be classified into lossy or lossless compression. It is the scaling of data set by quantization factor that results in irreversible data loss in quantization [32],[33],[27]. In the case of lossy compression, since all trivial samples are eliminated during quantization, higher compression ratios are obtained in comparison to lossless compression, but at the cost of the quality of the reconstructed image [33]

\subsection{Proposed S2ICAC Encoding Scheme}

In this section, a new compression model is proposed where the average pixel data of the two images is compressed and the disparity vector between the two images is compressed, which provides better compression results compared with other independent compression methods on individual images. The proposed Summative Stereoscopic Image Compression using Arithmetic Coding (S2ICAC) perform symmetric compression of the average image data and the disparity vector. The proposed new approach is designed for colour images though the algorithms work well for grayscale images.

This method achieved compression of stereoscopic images using the arithmetic coding by considering left and right view of an image. Since, the images are similar, encoding of average image and the differences between the two images provide higher compression rate. This approach exploits the correlation between the 3D-stereoscopic left image and right image. The approach is based on the observation that the encoding of the relative offset between the corresponding points in an image pair, termed as disparity leads to better compression. Hence, this approach exploits the correlation between the 3D-stereoscopic left image, right image, and a disparity vector mapping the dissimilarity between the two images are generated. In this approach, the colour space transformed average of left image and right image is first encoded by the arithmetic encoding and then the dissimilarity between the left and right images is also encoded by the arithmetic encoding. During decompression, the average image data is synthesized by application of arithmetic decoding. The right member is synthesized by performing inverse colour space transformation to the summated output obtained from decoded average image and arithmetically decoded disparity vector. The left member is synthesized by performing inverse colour space transformation to the variance output obtained from decoded average image and arithmetically decoded disparity vector. The proposed algorithm of S2ICAC Coding is shown below:

2.1.1. S2ICAC Compression Algorithm

1. Read the source left image, $\mathrm{L}$

2. Read source right image, $\mathrm{R}$

3. Perform the colour space conversion on $\mathrm{L}$ and obtain $\mathrm{CL}$

4. Perform the colour space conversion on $\mathrm{R}$ and obtain $\mathrm{CR}$

5. Perform average of CL and CR and obtain CA.

6. Perform Quantization on CA and obtain QCA

7. Obtain the disparity vector $\mathrm{D}$ from $\mathrm{CL}$ and $\mathrm{CR}$

8. Perform quantization on $\mathrm{D}$ and obtain QD

9. Perform Arithmetic Coding on the QCA and obtain AQCA

10. Perform Arithmetic Coding on the QD and obtain AQD

11. Compressed data size is noted

\subsubsection{S2ICAC Decompression Algorithm}

1. Read compressed data, AQCA

2. Read compressed data, AQD

3. Perform Arithmetic Decoding on AQCA and obtain retrieved average data, RICA

4. Perform Arithmetic Decoding on AQD and obtain retrieved RID 
5. Perform summation of RID and RICA to obtain RCR

6. Perform subtraction of RID from RICA to obtain RCL

7. Perform inverse colour space transformation on RCL and obtain retrieved left image RL

8. Perform inverse colour space transformation on RCR to obtain retrieved right image, RR

During the S2ICAC encoding process, the left source image, L, and right source image, R, are collected. Further, colour space conversion is performed on $\mathrm{L}$ and $\mathrm{R}$ to obtain $\mathrm{CL}$ and $\mathrm{CR}$. In the next stage, average data, CA is calculated by calculating the average of CL and CR. In the next stage, quantization is applied to CA and QCA is obtained. In the next stage, disparity vector, D is calculated by calculating the difference CL and CR. In the next stage, quantization is applied to D and QD is obtained. In the next stage, arithmetic coding is applied to QCA and QD and encoded data AQCA and AQD is generated.

During the decompression process, compressed data is read and the arithmetic decoding is applied to the AQCA and AQD, and retrieved data, RICA and RID is generated. In the next stage, summation of RID and RICA is performed to generate RCR and subtraction of RID from RICA is performed to generate RCL. Inverse colour transform is applied on RCR and RCL to generate the retrieved right image, RR and retrieved left image, RL respectively. The following Figure 1 shows flowchart of S2ICAC compression scheme.

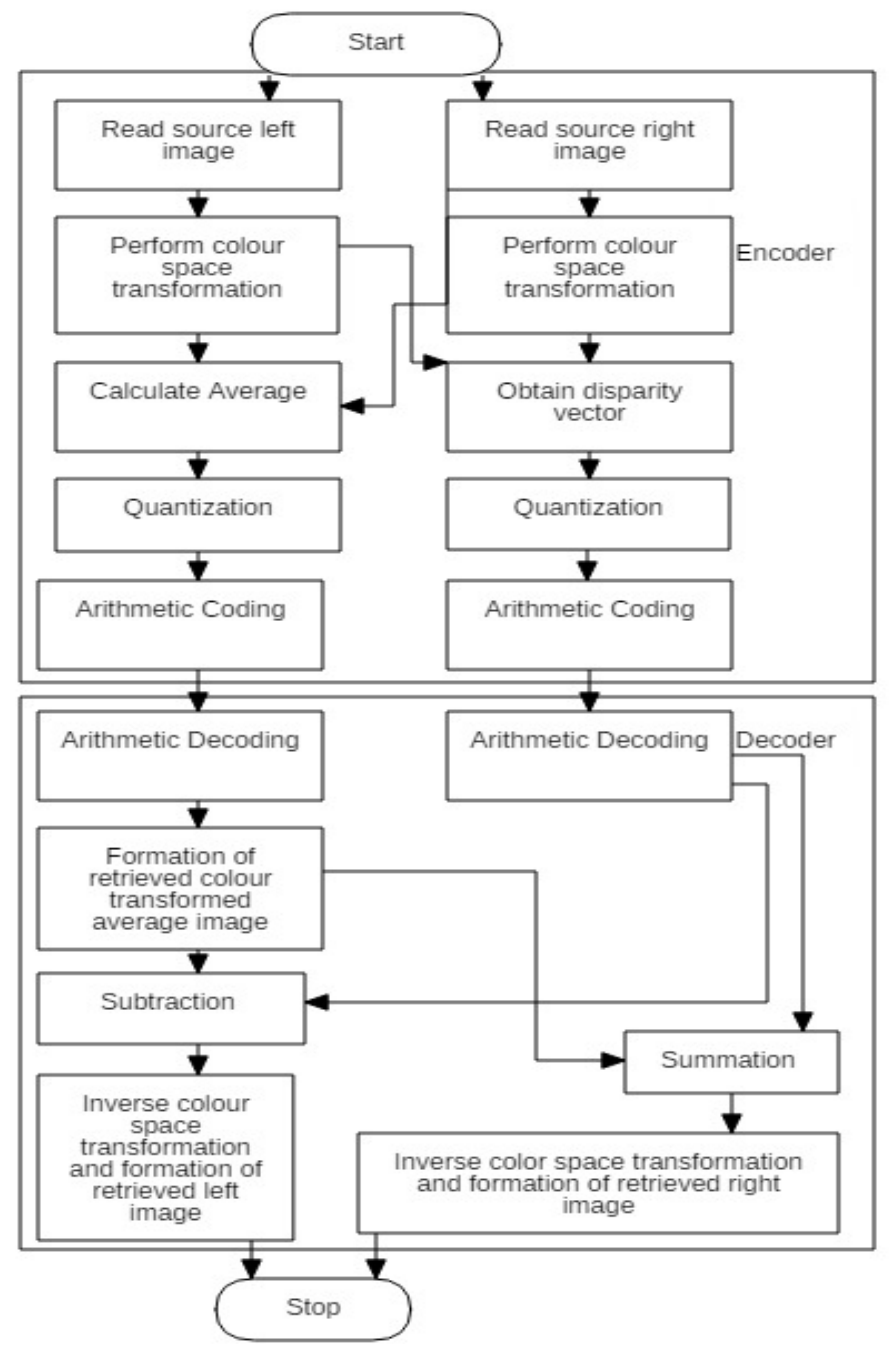

Figure 1. Block Diagram of proposed S2ICAC

Since the left image and right images of the stereopair are summated to obtain the average data, the proposed approach is coined as Summative Stereoscopic Image Compression using Arithmetic Coding (S2ICAC). Here, S2ICAC compression scheme is broadly classified as lossy S2ICAC compression and lossless S2ICAC compression. In the case of lossy S2ICAC compression, a close match of the original image is produced whereas in lossless compression, exact original image is reconstructed. In the case of lossless S2ICAC compression, there is no loss of data, whereas in lossy compression, some data is lost. In the proposed lossy S2ICAC method, the quantization steps result in the loss of data and the scheme is referred as lossy

Summative Stereoscopic Image Compression using Arithmetic Coding (TK Poolakkachalil, et al) 
S2ICAC. The scaling of data set by quantization factor is the cause for irreversible data loss in quantization. In the case of lossless S2ICAC, quantization is eliminated. In lossless approach, the reproduced image retains pixels value of the source image. CR is calculated from the arithmetic encoded value of the average and difference image. PSNR is calculated using from the outputs namely, retrieved left image, RCL and retrieved right image, RCR using equation 2.

An illustration of the methodology is explained below. For example, part of the source left image matrix is considered as $\mathrm{L}$ and part of source right image is considered as $\mathrm{R}$.

$\mathrm{L}=$

$\begin{array}{lllll}86 & 85 & 85 & 81 & 85 \\ 83 & 87 & 87 & 97 & 97 \\ 93 & 93 & 87 & 86 & 89 \\ 93 & 89 & 90 & 90 & 94 \\ 95 & 93 & 90 & 86 & 93\end{array}$

$\mathrm{R}=$

$\begin{array}{lllll}93 & 91 & 91 & 93 & 92 \\ 87 & 90 & 89 & 93 & 99 \\ 93 & 97 & 89 & 91 & 91 \\ 94 & 97 & 92 & 93 & 97 \\ 94 & 93 & 96 & 90 & 89\end{array}$

The following CL and CR represents the colour space transformed Y components of above L and R respectively. For illustrative purpose, one of the colour space components of $\mathrm{L}$ and $\mathrm{R}$ are shown below.

$\mathrm{CL}=$

$\begin{array}{ccccc}64.2203 & 63.3615 & 63.3615 & 60.4303 & 64.5655 \\ 63.3520 & 65.5833 & 63.8751 & 72.2675 & 72.2675 \\ 68.7343 & 69.4342 & 65.5833 & 64.2203 & 65.9989 \\ 68.8322 & 65.5927 & 66.2557 & 67.3619 & 71.3992 \\ 72.2580 & 70.5404 & 68.1597 & 64.6265 & 70.6383\end{array}$

$\mathrm{CR}=$

$\begin{array}{ccccc}70.4425 & 68.2207 & 67.2124 & 69.3363 & 70.0878 \\ 64.7854 & 67.2640 & 65.2990 & 68.7343 & 72.9915 \\ 68.0343 & 71.2738 & 65.8031 & 67.4229 & 67.3249 \\ 70.5035 & 71.2738 & 68.1838 & 70.6529 & 72.6737 \\ 71.1055 & 69.2384 & 71.9128 & 66.6619 & 65.7052\end{array}$

The following D represents the disparity vector obtained from CL and CR

$\mathrm{D}=$

$$
\begin{array}{ccccc}
-6.2222 & -4.8592 & -3.8510 & -8.9060 & -5.5222 \\
-1.4334 & -1.6807 & -1.4239 & 3.5332 & -0.7240 \\
0.6999 & -1.8396 & -0.2199 & -3.2026 & -1.3260 \\
-1.6713 & -5.6811 & -1.9281 & -3.2910 & -1.2745 \\
1.1525 & 1.3020 & -3.7531 & -2.0354 & 4.9331
\end{array}
$$

The following CA represents the average data obtained from CL and CR

$\begin{array}{llllll}\mathrm{CA}= & 67.3314 & 65.7911 & 65.28695 & 64.8833 & 67.3266 \\ 64.0687 & 66.4236 & 64.58705 & 70.5009 & 72.6295 \\ 68.3843 & 70.354 & 65.6932 & 65.8216 & 66.6619 \\ 69.6678 & 68.4332 & 67.2197 & 69.0074 & 72.0364 \\ & 71.6817 & 69.8894 & 70.0362 & 65.6442 & 68.1717\end{array}$

In the next stage, quantization is applied to $\mathrm{CL}$ and $\mathrm{D}$. The following QCA represents the quantized CA values.

$\mathrm{QCA}=$

$\begin{array}{lllll}68 & 60 & 60 & 60 & 68 \\ 60 & 68 & 60 & 70 & 70 \\ 68 & 70 & 60 & 60 & 68 \\ 68 & 68 & 68 & 68 & 70 \\ 70 & 68 & 70 & 60 & 68\end{array}$


The quantized D data, QD is generated in the same method. In the case of lossless S2ICAC, quantization process is eliminated.Further, arithmetic encoding is applied to QCA and QD and the encoded data AQCA and AQD is obtained.

During decompression, arithmetic decoding is applied to the encoded data, RICA, and disparity vector, RID is retrieved. Here, RICA is the retrieved colour transformed average image data. Following is an illustration of RICA and RID.

$\begin{array}{llllll}\mathrm{RICA}= & 76 & 84 & 92 & 76 & 76 \\ 68 & 68 & 84 & 84 & 84 \\ 68 & 68 & 76 & 84 & 92 \\ & 76 & 84 & 84 & 76 & 92 \\ 100 & 100 & 108 & 108 & 100\end{array}$

$\mathrm{RID}=$

$\begin{array}{lllll}4 & 12 & 12 & 4 & 4 \\ 4 & 4 & 4 & 4 & 4 \\ 4 & 4 & 4 & 4 & 4 \\ 4 & 4 & 4 & 4 & 4 \\ 4 & 4 & 4 & 4 & 4\end{array}$

Retrieved colour transformed right image data, RCR is obtained by the summation of RICA and RD. The following RCR represents the summated result of RICA and RID.

$\mathrm{RCR}=$

$\begin{array}{ccccc}80 & 96 & 104 & 80 & 80 \\ 72 & 72 & 88 & 88 & 88 \\ 72 & 72 & 80 & 88 & 96 \\ 80 & 88 & 88 & 80 & 96 \\ 104 & 104 & 112 & 112 & 104\end{array}$

Retrieved colour transformed leftimage data, RCR is obtained by the subtraction of RID from RICA. The following RCL represents the subtracted result of RICA and RID.

$\mathrm{RCL}=$

$\begin{array}{ccccc}72 & 72 & 80 & 72 & 72 \\ 64 & 64 & 80 & 80 & 80 \\ 64 & 64 & 72 & 80 & 88 \\ 72 & 80 & 80 & 72 & 88 \\ 96 & 96 & 104 & 104 & 96\end{array}$

In the next stage, inverse colour transform is applied to RCL and RCR. The following RL and RR represents the retrieved left image and retrieved right image obtained after application of inverse colour transform.

$\mathrm{RL}=$

$\begin{array}{lllll}84 & 84 & 84 & 84 & 84 \\ 84 & 84 & 84 & 84 & 84 \\ 84 & 92 & 84 & 84 & 84 \\ 84 & 84 & 84 & 84 & 84 \\ 84 & 84 & 92 & 84 & 84\end{array}$

$\mathrm{RR}=$

$\begin{array}{lllll}88 & 88 & 88 & 88 & 88 \\ 88 & 88 & 88 & 88 & 88 \\ 96 & 104 & 96 & 88 & 88 \\ 88 & 88 & 88 & 88 & 88 \\ 88 & 88 & 96 & 88 & 96\end{array}$

\section{RESULTS AND ANALYSIS}

In this section, we discuss the experimental results. S2ICAC is tested with a set of standard color images. Table 1 shows the original images used for testing and retrieved images with the two different proposed models. The simulations are performed on the popular LIVE 3D image quality database of the University of Texas, Austin, USA available at http://live.ece.utexas.edu/research/quality/live 3dimage phase1.html. The experiments are conducted on images with a dimension of $360 \times 640$. 
Table 1. Original images and retrieved images

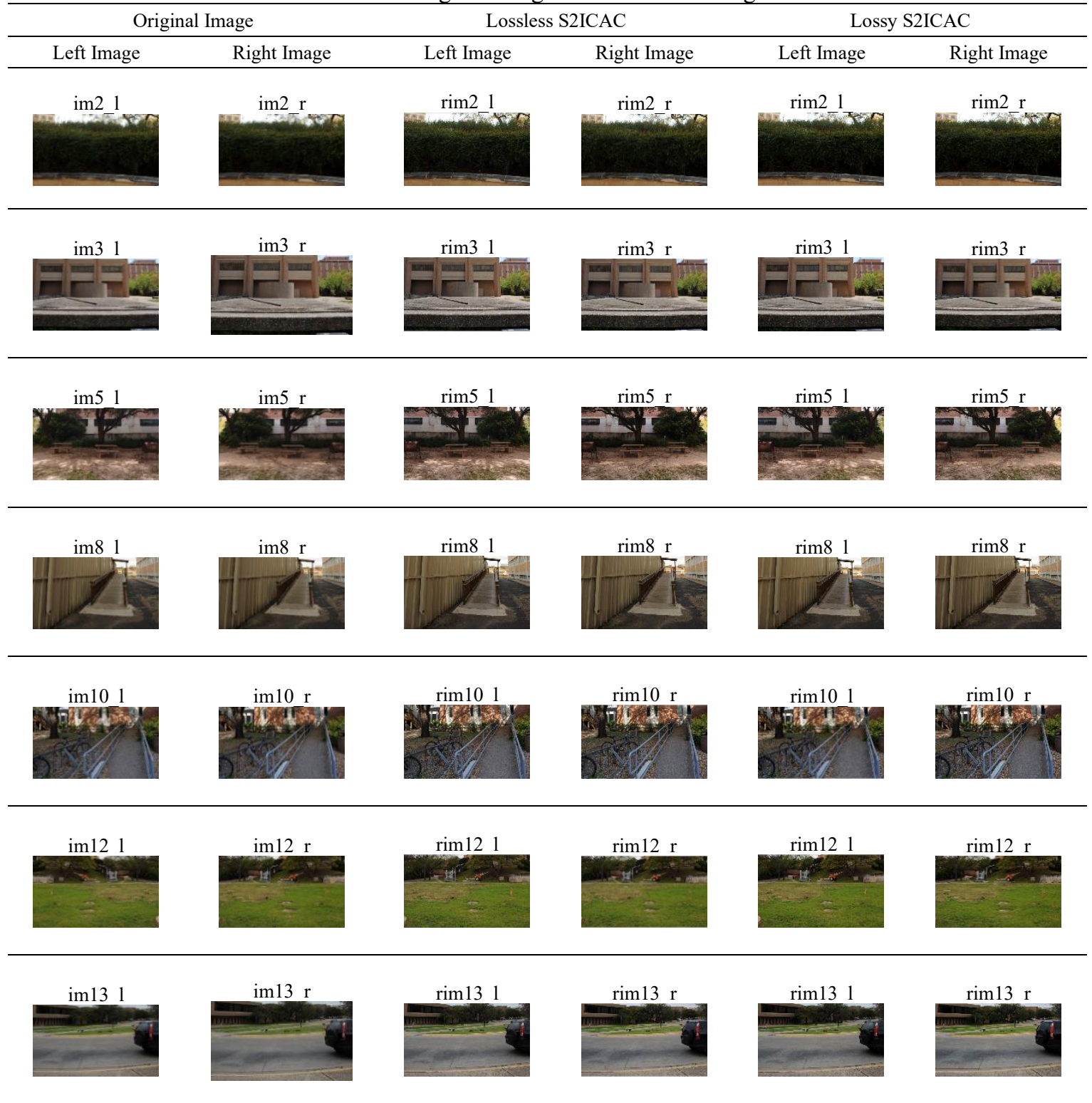

The following Table 2 shows the lossy compression ratio, lossless compression ratio, and respective PSNR values of the test images and their average using the proposed S2ICAC image compression scheme. It is observed from the experimental results that the highest CR under lossy S2ICAC scheme is for the image im13 and equals 23.9709 while the highest CR under lossless S2ICAC scheme is for the image im 3 and equals 13.0658. It is also observed that the highest PSNR is for im12 which equals 39.0745 under the lossy S2ICAC scheme while under the lossless S2ICAC scheme, the highest PSNR is also for im12 and equals 40.0165. The average CR and PSNR under the lossy S2ICAC scheme is 21.9991 and 33.3814 while the average CR and PSNR under the lossless S2ICAC scheme is 12.4884 and 34.2688.

Table 2. Compression Ratio (lossy and lossless) and PSNR using the proposed S2ICAC.

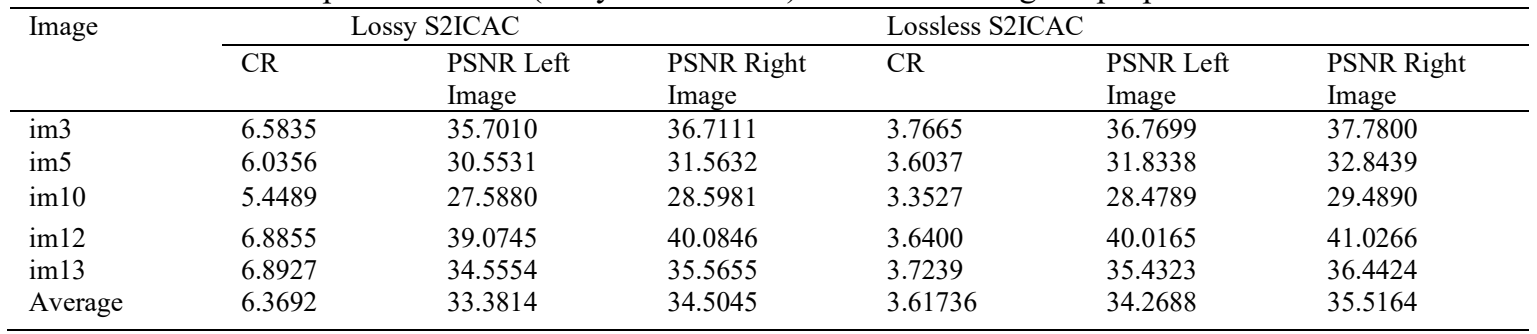


The comparison of CR under lossy S2ICAC and lossless S2ICAC is revealed in Figure 2. The results show that $\mathrm{CR}$ achieved under lossy S2ICAC is more than twice than that of CR achieved under lossless S2ICAC. Moreover, Figure 3 shows the comparison of PSNR of left image under lossy S2ICAC and lossless S2ICAC, while Figure 4 shows the comparison of PSNR of right image under lossy S2ICAC and lossless S2ICAC. It is observed that PSNR of left image and right image achieved under lossy S2ICAC is similar to that of PSNR achieved under lossless S2ICAC. From the above analysis, it is observed that lossy S2ICAC outperforms lossless S2ICAC.

The following Table 3 shows the compression results, PSNR of left and right images with respect to JPEG2000 with Position Based Coding Scheme [46].

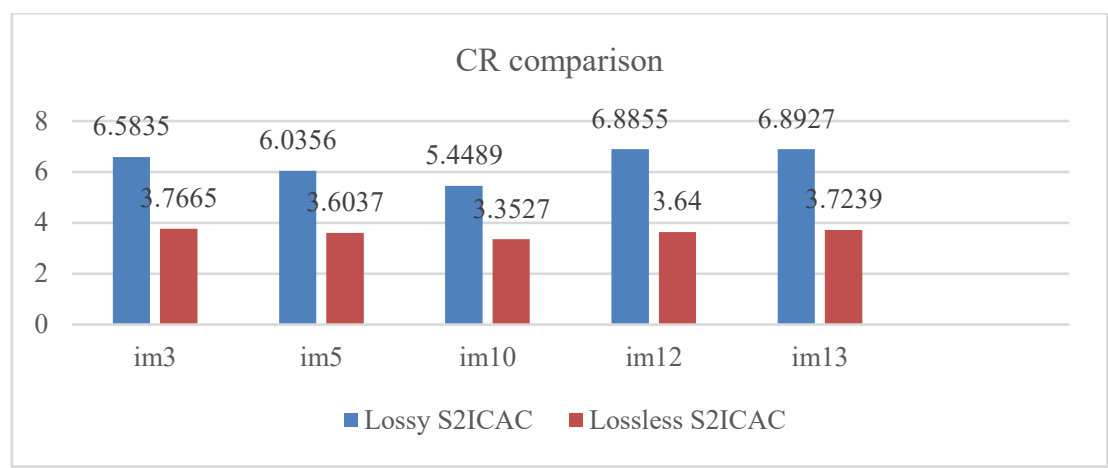

Figure 2. comparison of CR under lossy S2ICAC and lossless S2ICAC

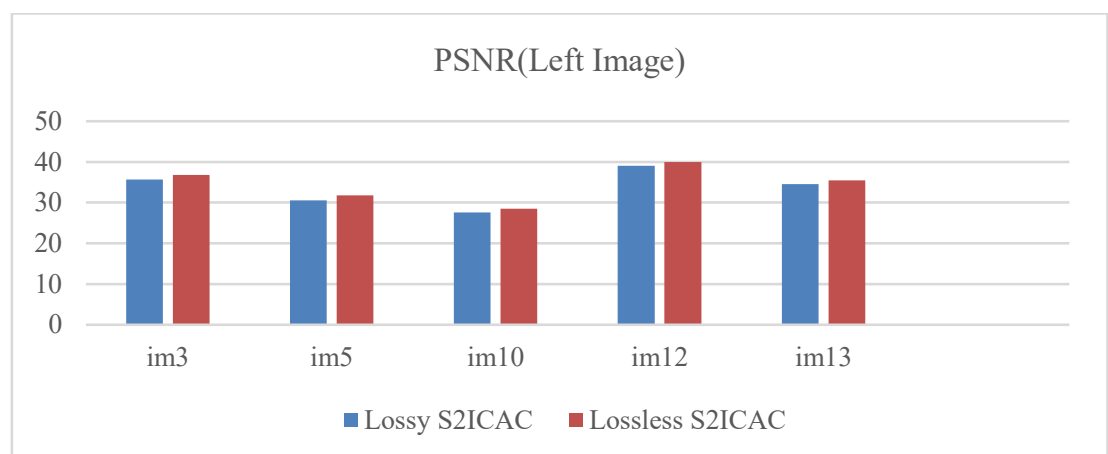

Figure 3. comparison of PSNR(Left Image) under lossy S2ICAC and lossless S2ICAC

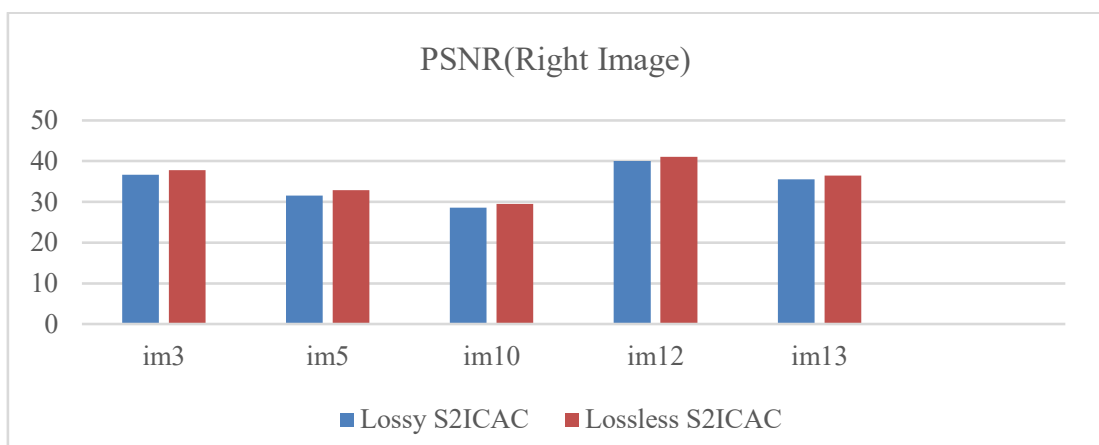

Figure 4. comparison of PSNR(Right Image) under lossy S2ICAC and lossless S2ICAC

Table 3. Compression Ratio and PSNR using JPEG2000 PBCS

\begin{tabular}{ccc}
\hline CR & PSNR Left Image & PSNR Right Image \\
\hline 3.2858 & 36.1280 & 36.1329 \\
3.2727 & 36.1247 & 36.1266 \\
3.2711 & 36.1274 & 36.1242 \\
3.3822 & 36.1203 & 36.1200 \\
3.3813 & 36.1232 & 36.1232 \\
3.2858 & 36.1280 & 36.1329 \\
\hline
\end{tabular}


Table 4 shows the compression results, PSNR of left and right images with respect to Stereoscopic Image Compression using Huffman Coding (SICHC)[47]. Moreover, Table 5 shows the comparison of average PSNR using proposed S2ICAC image compression scheme, JPEG 2000 Position Based Coding Scheme (JPEG 2000 PBCS) and Stereoscopic Image Compression using Huffman Coding (SICHC). From the results, it is observed that S2ICAC achieve higher compression ratio and higher quality compared with the JPEG 2000 PBCS and SICHC.

Table 4. Compression Ratio and PSNR using SICHC

\begin{tabular}{ccc}
\hline CR & PSNR Left Image & PSNR Right Image \\
\hline 4.3409 & 48.9400 & 48.9991 \\
4.7040 & 50.2012 & 47.1909 \\
3.9222 & 49.9553 & 46.9450 \\
4.3409 & 50.5210 & 47.5107 \\
4.9134 & 52.2717 & 49.2614 \\
4.3409 & 48.9400 & 48.9991 \\
\hline
\end{tabular}

Table 5. Comparison of PSNR of S2ICAC and JPEG 2000 PBCS

\begin{tabular}{cccc}
\hline Method & S2ICAC & JPEG 2000 PBCS & SICHC \\
\hline CR & $\mathbf{6 . 3 6 9 2}$ & 3.31862 & 4.42705 \\
PSNR Left Image & $\mathbf{3 3 . 3 8 1 4}$ & 36.1253 & 50.1382 \\
MSE Left Image & $\mathbf{1 7 . 6 9 7 8}$ & 16.8999 & 0.61398 \\
PSNR right Image & $\mathbf{3 4 . 5 0 4 5}$ & 36.1266 & 48.1510 \\
MSE Right Image & $\mathbf{1 5 . 6 9 8 5}$ & 16.5689 & 1.05974 \\
\hline
\end{tabular}

\section{CONCLUSION}

In this research article, a new compression model for stereoscopic image compression is proposed based on average and difference of the stereoscopic images. It is observed from the analysis of the results that the average CR with lossy S2ICAC is far more when compared to the average CR with lossless S2ICAC, while the PSNR values are comparable. This proved that lossy S2ICAC method is a better choice when compared to the lossless S2ICAC. This method is also compared with JPEG 2000 PBCS and SICHC. It is observed from the experimental results that the CR obtained by lossy S2ICAC is twice than that obtained using JPEG 2000 PBCS. It is also observed that CR achieved through lossy S2ICAC is better when compared to that of the CR achieved through SICHC. It is also observed that lossy S2ICAC exhibited good PSNR thus proving that quality of the image is preserved even with high CR in this approach.

\section{REFERENCES}

[1] L. Bondi, P. Bestagini, F. Pérez-González, S. Tubaro, "Improving PRNU Compression Through Preprocessing, Quantization, and Coding," IEEE Trans Inf Forensics Secur. 2019;14(3):608-20.

[2] E. Selmanovic, K. Debattista, T. Bashford-Rogers, A. Chalmers, "Enabling stereoscopic high dynamic range video," Signal Process Image Commun [Internet]. 2014;29(2):216-28. Available from: http://dx.doi.org/10.1016/j.image.2013.08.004

[3] Mendiburu, Bernard, "3D TV and 3D Cinema: Tools and Processes for Creative Stereoscopy1," Mendiburu ; Bernard. 3D TV and 3D Cinema: Tools and Processes for Creative Stereoscopy. Focal Press; 2011. Focal Press; 2011.

[4] H. Urey, K. V. Chellappan, E. Erden, P. Surman, "State of the art in stereoscopic and autostereoscopic displays," In: Proceedings of the IEEE. 2011. p. 540-55.

[5] J. P. McIntire, P. R. Havig, E. E. Geiselman, "What is 3D good for? A review of human performance on stereoscopic 3D displays," SPIE defense, Secur sensing Int Soc Opt Photonics [Internet]. 2012;83830X-83830X. Available from: http://proceedings.spiedigitallibrary.org/proceeding.aspx?doi=10.1117/12.920017

[6] D. J. Getty, P. J. Green, "Clinical applications for stereoscopic 3-D displays," J Soc Inf Disp [Internet]. 2007;15(6):377. Available from: http://doi.wiley.com/10.1889/1.1828693

[7] S. Dixon, E. Fitzhugh, D. Aleva, Human factors guidelines for applications of $3 D$ perspectives: a literature review. 2009;7327:73270K. Available http://proceedings.spiedigitallibrary.org/proceeding.aspx?doi=10.1117/12.820853

[8] J. J. Gallimore, "Visualization of Three-Dimensional Structure During Computer-Aided Design," Int J Hum Comput Interact. 1995;7(1):37-56.

[9] Y. C. Fan, Y. T. Kung, B. L. Lin. "Three-dimensional auto-stereoscopic image recording, mapping and synthesis system for multiview 3D display," In: IEEE Transactions on Magnetics. 2011. p. 683-6. 
[10] S. Mahmoudpour, M. Kim, The effect of depth map up-sampling on the overall quality of stereopairs. Displays. 2016;43:9-17.

[11] X. Li, Q. Ruan, Y. Jin, G. An, R. Zhao. "Fully automatic 3D facial expression recognition using polytypic multiblock local binary patterns," Signal Processing. 2015;108:297-308.

[12] A. Vosoughi, V. Testoni, P. C. Cosman, L. B. Milstein, "Multiview coding and error correction coding for 3D video over noisy channels," Signal Process Image Commun. 2015;30:107-20.

[13] Y. Liu, J. Yang, R. Chu, "Objective evaluation criteria for shooting quality of stereo cameras over short distance," Radioengineering. 2015;24(1):305-13.

[14] Issue STHE. Special Issue on 3-D Media and Displays. 2011;99(4):536-9.

[15] H. Kim, J. Lee, "A study on the possibility of implementing a real-time stereoscopic 3D rendering TV system," Displays [Internet]. 2015;40:24-34. Available from: http://dx.doi.org/10.1016/j.displa.2015.05.001

[16] A. Ortis, F. Rundo, G. Di Giore, S. Battiato, "Adaptive compression of stereoscopic images," In: Lecture Notes in Computer Science (including subseries Lecture Notes in Artificial Intelligence and Lecture Notes in Bioinformatics). 2013. p. 391-9.

[17] W. Yuanqing, "Estimating the minimum redundancy in stereo image pair," Image Vis Comput. 2008;26(5):72530.

[18] M. Siegel, P. Gunatilake, S. Sethuraman, A. Jordan, "Compression of stereo image pairs and streams," In: Stereoscopic Displays and Virtual Reality Systems SPIE [Internet]. 1994. p. 258-68. Available from: http://spie.org/x648.html?product_id=173899

[19] K. Zeger, "Residual image coding for stereo image compression," Opt Eng [Internet]. 2003;2(1):182. Available from:

http://link.aip.org/link/OPEGAR/v42/i1/p182/s1\&Agg=doi\%5Cnhttp://opticalengineering.spiedigitallibrary.org /article.aspx?articleid $=1099413$

[20] 76e73c84bc0cf4d5727e10270fd4b2592e50.pdf.crdownload.

[21] Fok S. Foveated Stereo Video Compression for Visual Telepresence by. 2002;

[22] R. Hawkins, Digital Stereo Video: display, compression and transmission. 2002;(February):1-148.

[23] M. S. Moellenhoff, M. W. Maier, "Transform coding of stereo image residuals," IEEE Trans Image Process. 1998;7(6):804-12.

[24] J. Konrad, Z. D. Lan. "Dense disparity estimation from feature correspondences," $\{$ Proc $\}\{$ Spie $\}\{$ Int $\}\{$ Soc $\}\{$ Opt $\}$ \{Eng\} [Internet]. 2000;3957:90-101. Available from: http://citeseerx.ist.psu.edu/viewdoc/download?doi=10.1.1.90.7029\&rep=rep1\&type=pdf

[25] I. N. G. A. Astaw, I. K. G. D. Putra, I. M. Sudarma, R. S. Hartati. "The Impact of Color Space and Intensity Normalization to Face Detection Performance," TELKOMNIKA (Telecommunication Comput Electron Control [Internet]. 2017;15(4):1894. Available from: http://journal.uad.ac.id/index.php/TELKOMNIKA/article/view/6718

[26] G. Sai, C. Kumar, R. Kiran Kumar, G. A. Naidu, J. Harikiran, "Noise Removal in Microarray Images Using Variational Mode Decomposition Technique," Telkomnika. 2017;15(4):1693-6930.

[27] R. C. Gonzalez, R. E. Woods, S. L. Eddins. "Digital Image Processing Using MATLAB." Vol. 1, Annals of Physics. 2004. 609 p.

[28] K. L. Chung, H. L. Huang, H. I. Lu, "Efficient region segmentation on compressed gray images using quadtree and shading representation," Pattern Recognit. 2004;37(8):1591-605.

[29] G. Zhai, W. Lin, J. Cai, X. Yang, W. Zhang, "Efficient quadtree based block-shift filtering for deblocking and deringing," J Vis Commun Image Represent. 2009;20(8):595-607.

[30] T. K. Poolakkachalil, S. Chandran, K. Vijayalakshmi, "Analysis of application of arithmetic coding on det and dct-dwt hybrid transforms of images for compression," In: 2017 International Conference on Networks and Advances in Computational Technologies, NetACT 2017. 2017.

[31] W-L Chen, Y-C Hu, K-Y Liu, C-C Lo, C-H Wen. "Variable-Rate Quadtree-segmented Block Truncation Coding for Color Image Compression,” Int J Signal Process Image Process Pattern Recognit [Internet]. 2014;7(1):6576. Available from: http://www.sersc.org/journals/IJSIP/vol7_no1/7.pdf

[32] P. G. K. Kharate, Color Image Compression Based On Wavelet Packet Best Tree. 2010;7(2):31-5.

[33] T. Koya Poolakkachalil, S. Chandran, Symbols Frequency based Image Coding for Compression, 2017;15(9):148-55.

[34] M. Siegel, S. Sethuraman, J. S. McVeigh, A. Jordan, "Compression and Interpolation of \{3D $\}$-Stereoscopic and Multi-View Video,” SPIE Stereosc Disp Virtual Real Syst IV. 1997;3012:227-38.

[35] A. Said, W. Pearlman, "A new, fast, and efficient image codec based on set partitioning in hierarchical trees," Circuits Syst Video Technol IEEE Trans. 1996;6(3):243-50.

[36] K. Ahn, An efficient disparity estimation algorithm for stereoscopic image compression. 1997;165-72.

[37] D. Tzovaras, N. Grammalidis, S. Member, “Object-Based Coding of Stereo Image Sequences Using Joint 3-D Motion / Disparity Compensation," IEEE Trans Circuits Syst. 1997;7(2):312-27.

[38] Directional EDGSE. REGULARIZATION TECHNIQUE FOR DISPARITY. 1999;376-7.

[39] W. H. Kim, S. W. Ra, "Fast disparity estimation using geometric properties and selectivesample decimation for stereoscopic image coding," Consum Electron IEEE Trans. 1999;45(1):203-9.

[40] P. R. Chang, C. F. Lin, M. J. Wu. "Design of multicode CDMA systems for 3-D stereoscopic video over wireless ATM networks," IEEE Trans Veh Technol. 2000;49(2):334-56.

[41] F. Labonté, C. T. Le Dinh, J. Faubert, P. Cohen . "Spatiotemporal spectral coding of stereo image sequences," IEEE Trans Circuits Syst Video Technol. 1999;9(1):144-55. 
[42] S. N. Sulthana, M. Chandra, Image Compression with Adaptive Arithmetic Coding. 2010;1(18).

[43] K. Muhammad, M. Sajjad, I. Mehmood, S. Rho, S. W. Baik, "A novel magic LSB substitution method (M-LSBSM) using multi-level encryption and achromatic component of an image," Multimed Tools Appl. 2016;75(22):14867-93.

[44] Y. Liu, J. Yang, Q. Meng, Z. Lv, Z. Song, Z. Gao. "Stereoscopic image quality assessment method based on binocular combination saliency model," Signal Processing [Internet]. 2016;125:237-48. Available from: http://dx.doi.org/10.1016/j.sigpro.2016.01.019

[45] P. Saipetch, B. K. T. Ho, R. Panwar, M. Ma, and J. Wei, “Applying wavelet transforms with arithmetic coding to radiological image compression," IEEE Eng Med Biol Mag. 1995;14(587-593).

[46] D. S. Vrindavanam Jayavrinda, Chandran, K. V. K. Mahanti Gautam .” JPEG, JPEG2000 and PBCS based Image Compression: An Experimental Analysis," Int J Comput Appl. 2012;58(10):975-8887.

[47] T. Koya Poolakkachalil, S. Chandran, Analysis of Stereoscopic Image Compression Using Arithmetic Coding and Huffman Coding. 2018; 214 - 220.

[48] M. Hernandez-Cabronero, I. Blanes, F. Auli-Llinas, J. Serra-Sagrista, V. Sanchez, M. W. Marcellin. "MosaicBased Color-Transform Optimization for Lossy and Lossy-to-Lossless Compression of Pathology Whole-Slide Images," IEEE Trans Med Imaging. 2018;38(1):21-32.

[49] S. Zhu, Z. He, X. Meng, S. Member, Transform-domain A. "Compression-Dependent Transform-Domain Downward Conversion for Block-Based” IEEE Trans Image Process. 2018;27(6):2635-49.

[50] Y. C. Fan, J. L. You, J. H. Shen, C. H. Wang. "Luminance and Color Correction of Multiview Image Compression for 3-DTV System," IEEE Trans Magn. 2014;50(7).

[51] J. M. G. Aranda, M. G. Casquete, M. C. Cueto, J. N. Salmerón, F. G. Vidal. "Logarithmical hopping encoding: a low computational complexity algorithm for image compression," IET Image Process [Internet]. 2015;9(8):643-51. Available from: http://oa.upm.es/37459/

[52] J. Zhang, H. Li, C. W. Chen, "Distributed Lossless Coding Techniques for Hyperspectral Images," IEEE J Sel Top Signal Process. 2015;9(6):977-89.

[53] P. Peter, L. Kaufhold, J. Weickert, "Turning diffusion-based image colorization into efficient color compression," IEEE Trans Image Process. 2017;26(2):860-9.

[54] R. Lan, Y. Zhou, Y. Y. Tang, "Quaternionic local ranking binary pattern: A local descriptor of color images," IEEE Trans Image Process. 2016;25(2):566-79.

[55] A. M. Raid, W. M. Khedr, M. A. El-dosuky, W. Ahmed. "Jpeg Image Compression Using Discrete Cosine Transform - A Survey," Int J Comput Sci Eng Surv [Internet]. 2014;5(2):39-47. Available from:http://www.airccse.org/journal/ijcses/papers/5214ijcses04.pdf 